\title{
Automatic consultation system for patients with cardiac implantable electronic devices undergoing magnetic resonance imaging
}

\author{
Donghoon Han ${ }^{1}$, Ji Hyun Lee ${ }^{1}$,I-Young Oh¹ In-ho Chae ${ }^{1}$, Ho-Young Lee ${ }^{2}$ and Youngjin Cho ${ }^{{ }^{*}}$ (c)
}

\begin{abstract}
Background: Safety evaluation for patients with cardiac implantable electronic devices (CIEDs) undergoing magnetic resonance imaging (MRI) scanning is often overlooked. We developed an automatic consultation system (ACS) to improve the screening rate in these patients.

Methods: ACS was developed by the Hospital Information System Development Department of Seoul National University Bundang Hospital. It was designed to automatically request pre-MRI cardiac evaluation in patients with CIED when MRI orders are issued. The proportion of the patients without pre-MRI cardiologic evaluation was evaluated before and after the ACS application.

Results: From January 2016 to June 2018, a total of 157 patients with CIEDs [pacemaker 136 (86.6\%), ICD or CRT-D 21 (13.4\%), MR-conditional 117 (74.5\%)] visited the MRI facility. Before the ACS application, 23 out of 84 patients (27.4\%) did not have adequate pre-MRI cardiologic evaluation. Despite urgent request for pre-MRI cardiac evaluation, MRI examination was postponed or cancelled in 14 (60.8\%) cases. After the ACS application, all 73 patients underwent proper cardiologic evaluation before their MRI examinations $(P<0.001)$. The proportion of immediate request for pre-MRI evaluation at the moment of MRI order also improved with the ACS application (before ACS 57.1\%, after ACS $100 \%, P<0.001)$.
\end{abstract}

Conclusions: The newly developed ACS helped the patients with CIED receive MRI scan safely on the schedule, improving the quality of care in this population.

Keywords: Hospital information system, Automatic consultation system, Cardiac implantable electronic device, Magnetic resonance imaging

\section{Introduction}

The number of patients with cardiac implantable electronic devices (CIEDs) is increasing, and many of these patients have various comorbidities [1,2]. A previous study reported that these patients have an approximately

*Correspondence: cho_y@snubh.org

${ }^{1}$ Division of Cardiology, Department of Internal Medicine, Seoul National University Bundang Hospital, Seoul National University College of Medicine, 82, Gumi-ro 173 beon-gil, Bundang-gu, Seongnam-si, Gyeonggi-do, Republic of Korea

Full list of author information is available at the end of the article
$75 \%$ chance of being indicated for a magnetic resonance imaging (MRI) scan over the lifetime of the device [3]. The prerequisite of the protocols for safe MRI scans in these patients $[4,5]$ is that the patient should be evaluated by experts in cardiology department before MRI scanning, which is often overlooked in daily clinical situations. To address this problem, we developed a new feature in our hospital information system (HIS) which provides automatic consultation to the cardiology department at the moment of issuing an MRI scan order in patients with CIED and investigated its beneficial effect. 


\section{Materials and methods Study population}

The patients with pacemakers or implantable cardioverter defibrillators who visited the MRI facility for MRI scan between January 2016 and June 2018 in Seoul National University Bundang Hospital (SNUBH) were consecutively enrolled. This study period consists of two parts: (1) before the application of the new automatic consultation system (ACS) (January 2016 to April 27, 2017) and (2) after the application of the ACS (from April 28,2017 , to June 2018). The development of the new system for patients with CIED undergoing MRI scan was one of the hospital's innovative projects, and during this period, patients with CIED who visited the MRI facility were prospectively listed up at both the MRI facility and the CIED analysis laboratory to evaluate the effects of the new system. Patients having either MR-conditional or conventional CIED who visited the MRI facility were enrolled in this study. This study protocol was approved by the Institutional Review Board of Seoul National University Bundang Hospital (B-1809-493-107) and was performed in accordance with the tenets of the Declaration of Helsinki.

\section{Automatic consultation system in the hospital information system}

The SNUBH developed its own hospital information system (HIS) named BESTCare, "Bundang hospital
Electronic System for Total Care" and upgraded it to the next-generation HIS, BESTCare 2.0 in 2013 [6, 7]. The project team consisting of cardiologists, nurses from cardiology department, MRI technicians and HIS department of SNUBH designed and developed an automatic consultation process into the HIS to improve quality and safety of the patients with CIEDs undergoing MRI scan on 2016. The hospital HIS department which had developed BESTCare 2.0 also developed this new ACS.

The scheme of the newly developed ACS is presented in Figs. 1 and 2. On the patient information window of the HIS, a new alert icon, "Device", was added to reveal information regarding implanted devices of the patient. Inside the "Device" alert icon, "CIED" checkbox was designed to be checked automatically when the orders regarding CIED implantation surgery or interrogation were detected in the SNUBH HIS (BESTCare 2.0). In patients with activated "CIED" checkbox, a red shoulder badge appears on "Device" alert icon (Fig. 1). This alert sign can be activated manually, for exceptional cases such as a patient who implanted CIED elsewhere and visits our hospital for an MRI evaluation. When an MRI order is issued in a patient with an activated "Device" alert, a warning pop-up window automatically opens. This warning window not only notifies the ordering physician that the patient needs to be checked for the MRI safety of the CIED, but also asks whether to make a consultation request to a cardiologist. Simply clicking "yes" opens a

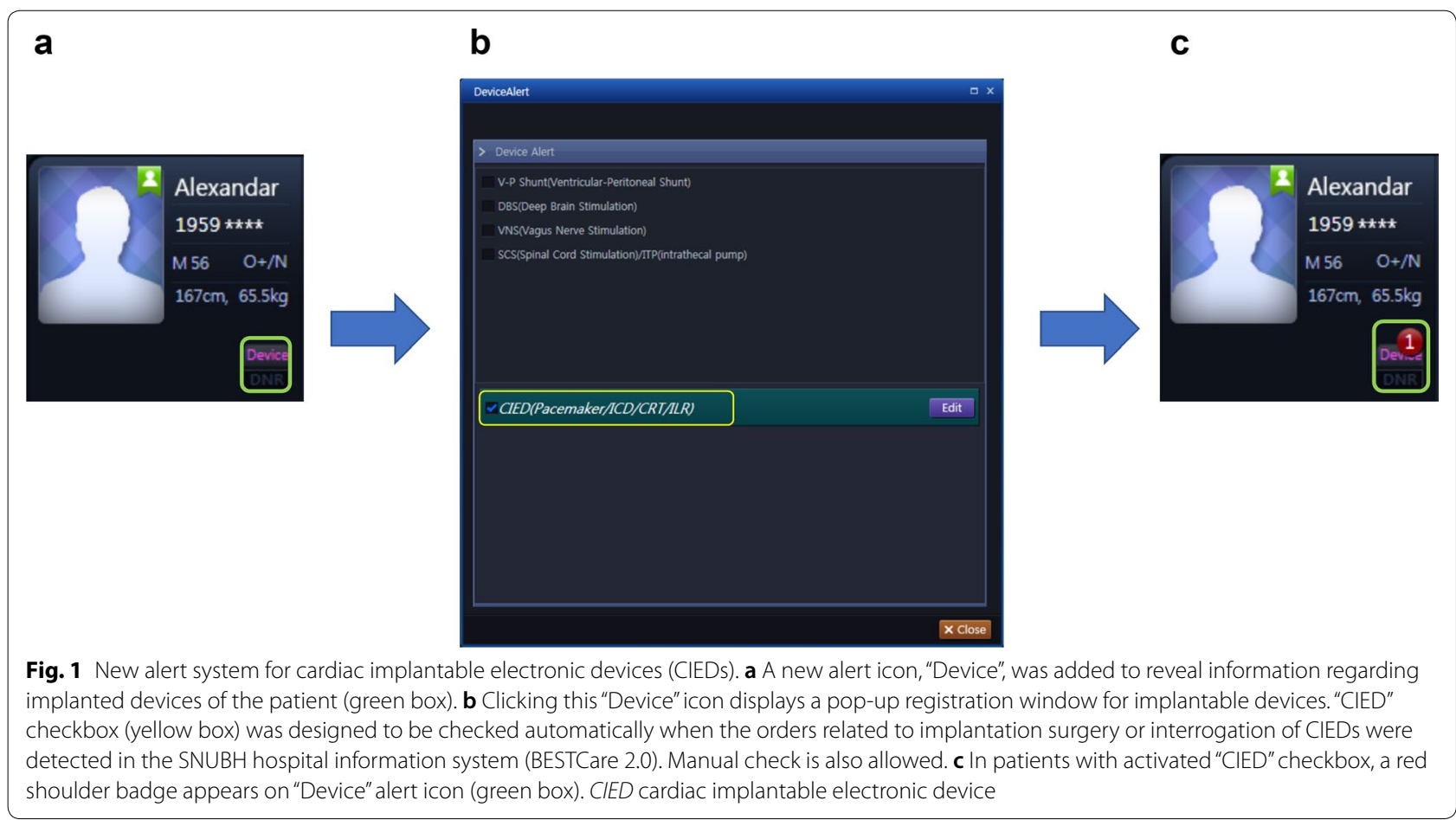




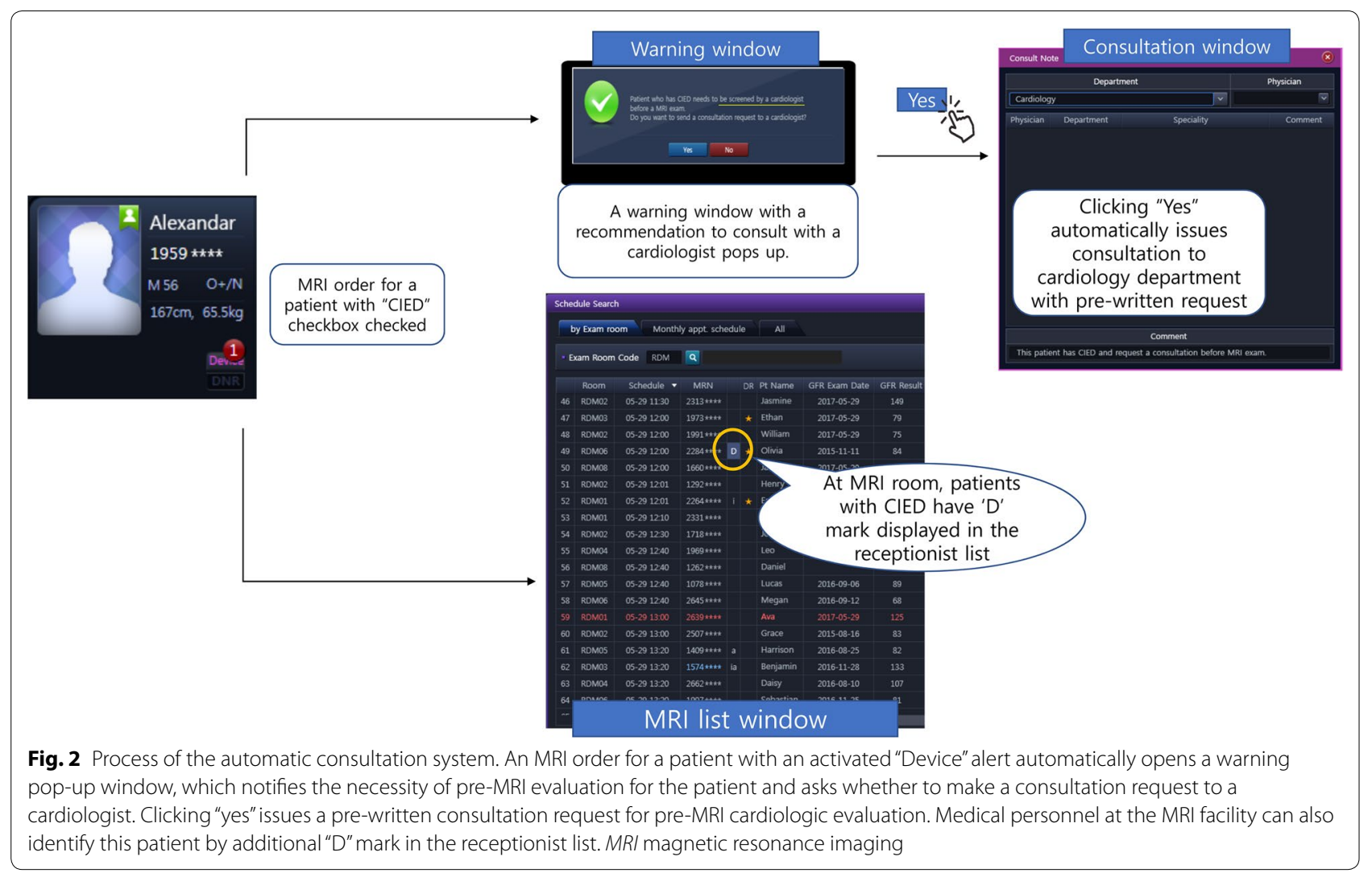

consultation request window with a pre-written request for a safety check of CIEDs under MRI scan. Then, a visit to the cardiology department would be arranged on the day of MRI, allowing the patient to perform device interrogation and required changes in CIED modes before and after MRI scan. In the MRI room, "Device" alert mark also can be screened on the patient's registration window, warning the radiologists and technicians to monitor the patients with caution (Fig. 2). After examination, 1-month follow-up visit to cardiology department is reserved. We completed development of this system and applied it in the clinical field on April 28, 2017.

\section{Effects of the automatic consultation system}

We evaluated the beneficial effects of the ACS as the proportion of the adequate cardiology consultation before the patients with CIED visit the MRI facility. In addition, it was investigated whether pre-MRI consultation to cardiology department was requested immediately at the time of MRI order or later after being screened by medical personnel in the MRI room. For this purpose, pre-MRI evaluation was considered to be requested immediately after MRI order if the consultation request was issued on the same day as the MRI order. In addition, cancellation rate was compared among the periods.

\section{Statistical analysis}

The Student's $t$ test and Chi-square (or Fisher's exact) test were used to compare means and proportion of baseline clinical characteristics between the groups. All of the statistical analyses were performed using $\mathrm{R}$ version 3.6.0, and $P<0.05$ was considered statistically significant.

\section{Results}

Characteristics of the study population

A total of 157 patients with CIEDs visited the MRI facility during the study period. There were 84 cases before and 74 cases after the ACS application. Clinical characteristics of the study population are presented in Table 1 . The mean age was $73.8 \pm 10.8$ before ACS and $75.1 \pm 10.2$ after ACS $(P=0.414)$. The proportion of MR-conditional devices, which are known to pose no known hazards in a specific MRI environment under specific MRI scanner condition [8], was not significantly different [before ACS 58/83 (70.7\%), after ACS 59/73 (80.8\%), $P=0.204]$. The proportions of high-voltage devices such as implantable cardioverter-defibrillator (ICD) or cardiac resynchronization therapy defibrillator (CRT-D) were 12/84 (14.3\%) and $9 / 73(12.3 \%)$, before and after ACS, respectively $(P=0.901)$. Among them, $9 / 12(75.0 \%)$ and $8 / 9(88.9 \%)$ were MR-conditional devices, respectively $(P=0.810)$. 
Table 1 Characteristics of the study population

\begin{tabular}{llll}
\hline & $\begin{array}{l}\text { Before ACS } \\
(\boldsymbol{n = 8 4 )}\end{array}$ & $\begin{array}{l}\text { After ACS } \\
(\boldsymbol{n = 7 3 )}\end{array}$ & P value \\
\hline Age (years) & $73.8 \pm 10.8$ & $75.1 \pm 10.2$ & 0.414 \\
Male & $49(58.3)$ & $35(47.9)$ & 0.254 \\
MR-conditional & $58(70.7)$ & $59(80.8)$ & 0.204 \\
Pacemaker & $72(85.7)$ & $64(87.7)$ & 0.901 \\
MR-conditional & $49(70.0)$ & $51(79.7)$ & 0.276 \\
Indications & & & 0.327 \\
$\quad$ AV block & $32(44.4)$ & $36(56.3)$ & \\
SSS & $40(55.6)$ & $28(43.7)$ & \\
ICD orCRT-D & $12(14.3)$ & $9(12.3)$ & 0.901 \\
MR-conditional & $9(75.0)$ & $8(88.9)$ & 0.810 \\
Indications & & & 0.331 \\
Primary prevention & $9(75.0)$ & $4(44.4)$ & \\
Secondary prevention & $3(25.0)$ & $5(55.6)$ & \\
MRl scan site & & & 0.014 \\
Brain & $48(73.8)$ & $37(50.7)$ & \\
Spine & $9(13.8)$ & $24(32.9)$ & \\
Others & $8(12.3)$ & $12(16.4)$ & \\
Emergency room & $12(14.5)$ & $8(11.0)$ & 0.680 \\
\hline Data & &
\end{tabular}

Data are expressed as $\mathrm{n}(\%)$ or mean $\pm \mathrm{SD}$

ACS automatic consultation system, $M R I$ magnetic resonance imaging, $A V$ atrioventricular, ICD implantable cardioverter-defibrillator, CRT-D cardiac resynchronization therapy defibrillator, SSS sick sinus syndrome

a Numbers may not sum to total due to cancellation of the examination after visiting the MRI facility or scanning of multiple sites

The most frequent MRI scan site was the brain in both groups. MRI was ordered in the setting of emergency in 20/157 (12.7\%) cases.

\section{Pre-MRI cardiology consultation}

The proportion of patients visiting the MRI facility with cardiologic evaluation before and after the ACS application is presented in Table 2. Before the setting of the ACS, the rates of adequate screening consultation to cardiology department before visiting MRI facility in patients with CIED were $72.6 \%$. After the ACS application, all 73 patients underwent pre-MRI cardiologic evaluation before MRI examination $(P<0.001)$. Regarding the timing of the request for pre-MRI evaluation, more patients were immediately referred to the cardiology department after the application of the new ACS $(P<0.001)$.

Patients without pre-MRI cardiology evaluation were screened at the MRI facility at the day of the examination and were sent to a cardiologist for screening evaluation, which resulted in postponing or cancellation of MRI examination in $14 / 23(60.8 \%)$ cases. With urgent requests, $9 / 23(29.2 \%)$ patients were able to be evaluated by a cardiologist and underwent MRI scan on the scheduled day. There was no patient with CIED visiting the MRI facility without pre-MRI cardiac evaluation.
Table 2 Changes in the proportion of patients with preMRI cardiologic evaluation after the application of ACS

\begin{tabular}{llll}
\hline & $\begin{array}{l}\text { Before ACS } \\
(\boldsymbol{n = 8 4 )}\end{array}$ & $\begin{array}{l}\text { After ACS } \\
(\boldsymbol{n}=\mathbf{7 3})\end{array}$ & P value \\
\hline $\begin{array}{l}\text { MRI visit with cardiologic evalu- } \\
\text { ation }\end{array}$ & $61(72.6)$ & $73(100)$ & $<0.001$ \\
$\begin{array}{l}\text { Evaluation requested immedi- } \\
\text { ately after MRI order }\end{array}$ & $48(57.1)$ & $73(100)$ & $<0.001$ \\
$\begin{array}{l}\text { MRI visit without cardiologic } \\
\text { evaluation }\end{array}$ & $23(27.4)$ & $0(0)$ & $<0.001$ \\
$\begin{array}{l}\text { MRI as scheduled after urgent } \\
\text { evaluation }\end{array}$ & $9(39.1)$ & - & - \\
$\begin{array}{l}\text { MRI rescheduled } \\
\text { MRI cancelled after evaluation } \\
\text { 1-month follow- } \text { - }\end{array}$ & $13(56.5)$ & - & - \\
\hline
\end{tabular}

Data are expressed as $n(\%)$

ACS automatic consultation system, MRI magnetic resonance imaging

After the ACS application, all patients underwent follow-up CIED interrogation 1 month after the MRI examination, and there was no evidence of MRI-related complications.

\section{Discussion}

MRI has become the imaging modality of choice in various clinical situations, and the use of MRI scans has markedly increased [9]. Also the number of patients with CIEDs is increasing [10]; thus, the patients with pacemakers and ICDs are often indicated for an MRI scan over the lifetime of the device [1-3]. Nazarian et al. [11] reported that more than $36 \%$ of patients with ICDs would require an MRI scan within 4 years of device implantation. Despite the potential risks of the interactions between the MRI environment and conventional CIEDs, the evidences supporting the safety of MRI in patients with conventional CIEDs are recently growing [12]. Several protocols were suggested to perform MRI scan safely, at least in the selected patients with selected CIEDs [4, 13-17]. Similarly, we have previously reported that MRI examinations could be performed without adverse events or significant parameter changes in both the MR-conditional and the conventional CIED groups [18]. Since the first FDA approval for the MR-conditional pacing devices in 2011, MR-conditional CIEDs have been rapidly replaced conventional non-MR-conditional devices in the market, reducing the concerns regarding the safety issues of MRI in patients with CIEDs. Nevertheless, to perform MRI safely, MR-conditional devices still require specific device programming, imaging protocols and also continuous monitoring during the MRI, similarly as conventional devices [19-22]. Therefore, pre-MRI evaluation by cardiologists is crucial for safe MRI examination in 
patients with CIEDs, regardless of whether their CIEDs are MR-conditional or not. This study introduces the newly developed automatic consultation system incorporated in the HIS, for patients with CIEDs undergoing MRI scan. The results suggest that this new system could improve the safety of MRI scans in this population.

Increased utilization of MRI in patients with CIEDs may be partly from expansion of MR-conditional CIEDs. During the past 5 years, the number of patients with CIEDs having MRI scanning in SNUBH has increased, mainly attributed by the increase in MRI cases with MRconditional CIEDs (Fig. 3). However, it should be empathized that high prevalence of MR-conditional CIEDs by itself cannot translate into better quality of care. In this study, more than one-fourth of the patients with CIED visited the MRI facility without adequate pre-MRI cardiac evaluation before the ACS application. MRI orders are frequently placed by non-cardiologists, and some physicians' misunderstanding that "MR-conditional" devices do not require any evaluations or changes in device programming before MRI scan may have led to this negligence of pre-MRI cardiologic screening. It is noteworthy because we promoted pre-MRI cardiology consultation to the medical personnel during this ACS developmental period. Therefore, this result also suggests that promotion and education alone would not be sufficient in achieving the safety of patients with CIED

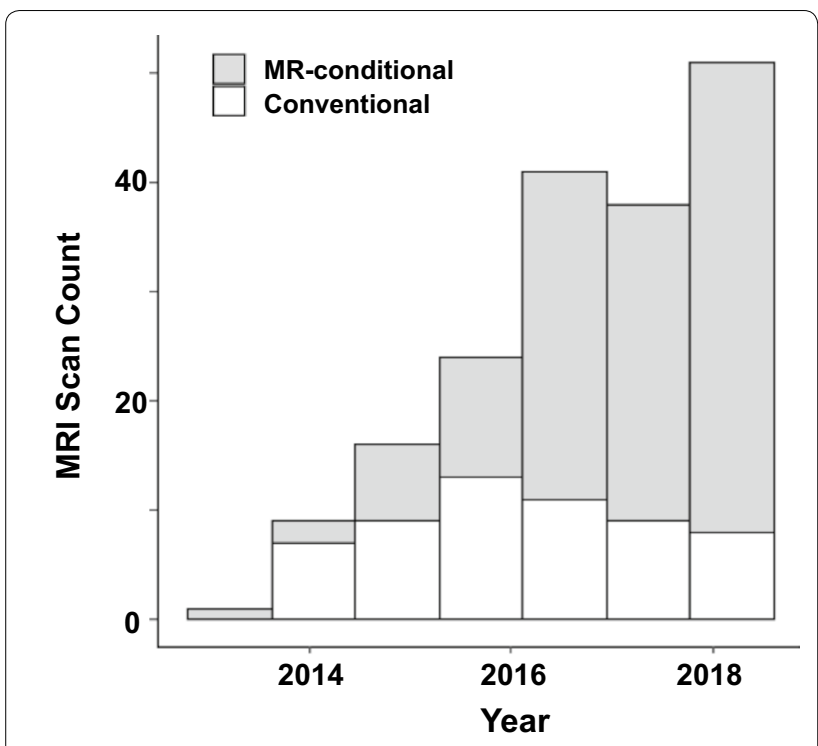

Fig. 3 Trend of MRI scan in the patients with CIEDs in the Seoul National University Bundang Hospital. The number of MRI scan in patients with CIED has gradually increased. The proportion of MR-conditional CIEDs among the patients underwent MRI scan has also increased. CIED cardiac implantable electronic device, MRI magnetic resonance imaging undergoing MRI. In this respect, systematic approach such as our new ACS may improve the quality of care in these populations.

Before a patient with CIED has an MRI scan, there are several points in time when the patient can be screened. A patient can be screened first when the MRI order is placed, when MRI examination is scheduled, when obtaining written consent for the examination and lastly when the MRI scan is actually performed. The first step, at the moment of issuing MRI scan order, was considered to be a good target to intervene, not only to improve the rate of pre-MRI cardiologic evaluation, but also to decrease the dissatisfaction of patients as a consequence of cancellation or postponing of the examination. Therefore, the main feature of this ACS was to generate an automatic consultation request at the moment of the MRI order placement. As an additional screening filter for these patients, the ACS was designed to alert medical personnel with activated alert icons that can be seen from the patient list of the MRI room, notifying that this patient has CIED. This feature provided another safety net to ensure appropriate preparation and monitoring before and during MRI examinations.

After adoption of the new ACS, all patients received MRI scan after adequate cardiology consultation, device interrogation and devices reprogramming. Moreover, all patients revisited the cardiology department for follow-up analysis of their implanted devices, and all patients received MRI scan on the schedule, without delays. Therefore, it is suggested that the application of the ACS has improved the patients' satisfaction, the safety of MRI examination and the quality of care in the study population.

This study is underpowered to assess the actual clinical effect following the increase in pre-MRI cardiologic consultations. There were no reports of significant MRIrelated adverse events during the study period regardless of ACS application, though it might have been underreported because some patients missed follow-up CIED interrogation before the ACS application period. Nevertheless, this study showed that the addition of a new alert process in the electronic hospital records system significantly increased the quality of care and helped avoid nearmiss events which may potentially lead to harmful results.

\section{Conclusions}

The newly developed ACS helped patients with pacemakers or implantable cardioverter defibrillators receive their MRI scan safely on schedule and visit the cardiology department for 1-month follow-up analysis of the devices. Utilization of this system could help improve the quality of care in patients with CIEDs undergoing MRI examinations. 


\section{Abbreviations}

ACS: automatic consultation system; CIED: cardiac implantable electronic device; CRT-D: cardiac resynchronization therapy defibrillator; HIS: hospital information system; ICD: implantable cardioverter-defibrillator; MRI: magnetic resonance imaging.

\section{Acknowledgements}

Not applicable.

\section{Authors' contributions}

$\mathrm{DH}$ acquired and analysed the data and drafted the manuscript. JHL analysed the data and substantively revised the manuscript. IYO contributed to the conception of the work and revised the manuscript. IC contributed the design of the work and revised the manuscript. HYL created an automatic consultation system used in the study. YC designed the work, analysed the data and revised the manuscript. All authors read and approved the final manuscript.

\section{Funding}

Not applicable.

\section{Availability of data and materials}

All data generated or analysed during this study are included in this published article.

\section{Ethics approval and consent to participate}

This study protocol was approved by the Institutional Review Board of Seoul National University Bundang Hospital (B-1809-493-107) and was performed in accordance with the tenets of the Declaration of Helsinki.

\section{Consent for publication}

Not applicable.

\section{Competing interests}

The authors declare that they have no competing interests.

\section{Author details \\ ${ }^{1}$ Division of Cardiology, Department of Internal Medicine, Seoul National University Bundang Hospital, Seoul National University College of Medicine, 82, Gumi-ro 173 beon-gil, Bundang-gu, Seongnam-si, Gyeonggi-do, Republic of Korea. ${ }^{2}$ Office of eHealth Research and Business, Seoul National University Bundang Hospital, 82, Gumi-ro 173 beon-gil, Bundang-gu, Seongnam-si, Gyeonggi-do, Republic of Korea.}

Received: 3 October 2019 Accepted: 20 December 2019

Published online: 22 January 2020

\section{References}

1. Michael Niehaus JRT. Electromagnetic interference in patients with implanted pacemakers or cardioverter-defibrillators. Heart. 2001;86:246-8.

2. Douglas J, Quint M. ndications for emergent MRI of the central nervous system. JAMA. 2000;283:853-5.

3. Kalin R, Stanton MS. Current clinical issues for MRI scanning of pacemaker and defibrillator patients. Pacing Clin Electrophysiol. 2005;28:326-8.

4. Nazarian S, Roguin A, Zviman MM, Lardo AC, Dickfeld TL, Calkins H, Weiss RG, Berger RD, Bluemke DA, Halperin HR. Clinical utility and safety of a protocol for noncardiac and cardiac magnetic resonance imaging of patients with permanent pacemakers and implantable-cardioverter defibrillators at 1.5 tesla. Circulation. 2006;114:1277-84.

5. Nazarian S, Halperin HR. How to perform magnetic resonance imaging on patients with implantable cardiac arrhythmia devices. Heart Rhythm 2009:6:138-43.

6. Yoo S, Lee KH, Lee HJ, Ha K, Lim C, Chin HJ, Yun J, Cho EY, Chung E, Baek RM, Chung CY, Wee WR, Lee CH, Lee HS, Byeon NS, Hwang H. Seoul National University Bundang Hospital's Electronic System for Total Care. Healthc Inform Res. 2012:18:145-52.

7. Yoo S, Hwang H, Jheon S. Hospital information systems: experience at the fully digitized Seoul National University Bundang Hospital. J Thorac Dis. 2016;8:S637-41.
8. Shellock FG, Woods TO, Crues JV 3rd. MR labeling information for implants and devices: explanation of terminology. Radiology. 2009;253:26-30.

9. Sutton R, Kanal E, Wilkoff BL, Bello D, Luechinger R, Jenniskens I, Hull M, Sommer T. Safety of magnetic resonance imaging of patients with a new Medtronic EnRhythm MRI SureScan pacing system: clinical study design. Trials. 2008;9:68

10. Greenspon AJ, Patel JD, Lau E, Ochoa JA, Frisch DR, Ho RT, Pavri BB, Kurtz SM. Trends in permanent pacemaker implantation in the United States from 1993 to 2009: increasing complexity of patients and procedures. J Am Coll Cardiol. 2012;60:1540-5.

11. Nazarian S, Reynolds M, Ryan M, Hunter T, Wolff S, Mollenkopf S, Turakhia M. Estimating the likelihood of MRI in patients after ICD implantation: a 10-year prediction model. J Am Coll Cardiol. 2015;65:1090.

12. The Task Force on Cardiac Pacing, Resynchronization Therapy of the European Society of Cardiology. Developed in collaboration with the European Heart Rhythm Association, Brignole M, Auricchio A, Baron-Esquivias G, Bordachar P, Boriani G, Breithardt OA, Cleland J, Deharo JC, Delgado V, Elliott PM, Gorenek B, Israel CW, Leclercq C, Linde C, Mont L, Padeletti L, Sutton R, Vardas PE. 2013 ESC guidelines on cardiac pacing and cardiac resynchronization therapy. Rev Esp Cardiol (Engl Ed). 2014;67:58.

13. Martin ET, Coman JA, Shellock FG, Pulling CC, Fair R, Jenkins K. Magnetic resonance imaging and cardiac pacemaker safety at 1.5-Tesla. J Am Coll Cardiol. 2004;43:1315-24.

14. Sommer T, Naehle CP, Yang A, Zeijlemaker V, Hackenbroch M, Schmiedel A, Meyer C, Strach K, Skowasch D, Vahlhaus C, Litt H, Schild H. Strategy for safe performance of extrathoracic magnetic resonance imaging at 1.5 tesla in the presence of cardiac pacemakers in non-pacemaker-dependent patients: a prospective study with 115 examinations. Circulation. 2006;114:1285-92.

15. Russo RJ, Costa HS, Silva PD, Anderson JL, Arshad A, Biederman RW, Boyle NG, Frabizzio JV, Birgersdotter-Green U, Higgins SL, Lampert R, Machado CE, Martin ET, Rivard AL, Rubenstein JC, Schaerf RH, Schwartz JD, Shah DJ, Tomassoni GF, Tominaga GT, Tonkin AE, Uretsky S, Wolff SD. Assessing the risks associated with $\mathrm{MRI}$ in patients with a pacemaker or defibrillator. $\mathrm{N}$ Engl J Med. 2017;376:755-64.

16. Roguin A, Zviman MM, Meininger GR, Rodrigues ER, Dickfeld TM, Bluemke DA, Lardo A, Berger RD, Calkins H, Halperin HR. Modern pacemaker and implantable cardioverter/defibrillator systems can be magnetic resonance imaging safe: in vitro and in vivo assessment of safety and function at 1.5 T. Circulation. 2004;110:475-82.

17. Roguin A. Magnetic resonance imaging in patients with implantable cardioverter-defibrillators and pacemakers. J Am Coll Cardiol. 2009;54:556-7.

18. Han D, Kang SH, Cho Y, Oh IY. Experiences of magnetic resonance imaging scanning in patients with pacemakers or implantable cardioverterdefibrillators. Korean J Intern Med. 2018;34:99.

19. Forleo GB, Santini L, Della Rocca DG, Romano V, Papavasileiou LP, Magliano G, Sgueglia M, Romeo F. Safety and efficacy of a new magnetic resonance imaging-compatible pacing system: early results of a prospective comparison with conventional dual-chamber implant outcomes. Heart Rhythm. 2010;7:750-4.

20. Gold MR, Sommer T, Schwitter J, Al Fagih A, Albert T, Merkely B, Peterson M, Ciuffo A, Lee S, Landborg L, Cerkvenik J, Kanal E, Evera MRISI. Full-body MRI in patients with an implantable cardioverter-defibrillator: primary results of a randomized study. J Am Coll Cardiol. 2015;65:2581-8.

21. Shinbane JS, Colletti PM, Shellock FG. Magnetic resonance imaging in patients with cardiac pacemakers: era of "MR Conditional" designs. J Cardiovasc Magn Reson. 2011;13:63.

22. Verma A, Ha AC, Dennie C, Essebag V, Exner DV, Khan N, Lane C, Leipsic J, Philippon F, Sampaio M, Schieda N, Seifer C, Berthiaume A, Campbell D, Chakraborty S. Canadian Heart Rhythm Society and Canadian Association of Radiologists consensus statement on magnetic resonance imaging with cardiac implantable electronic devices. Can J Cardiol. 2014:30:1131-41.

\section{Publisher's Note}

Springer Nature remains neutral with regard to jurisdictional claims in published maps and institutional affiliations. 